\title{
SÍNTESIS Y CARACTERIZACIÓN \\ DE COMPOSITOS A BASE DE HALLOYSITA \\ CON NANOESTRUCTURAS DE BISMUTO
}

\author{
SYNTHESIS AND CHARACTERIZATION OF COMPOSITES \\ BASED ON HALLOYSITE AND BISMUTH NANOESTRUCTURES
}

Carolina Vega-Verduga ${ }^{1,2^{*}}$ \& Caterine Daza-Gómez ${ }^{2}$

Recibido: 30 de septiembre 2019 / Aceptado: 18 de diciembre 2019

DOI: $10.26807 /$ ia.v8i1.124

Palabras clave: aglomeración, halloysita, nanocomposito, nanotubos, bismuto

Keywords: agglomeration, bismuth, halloysite, nanocomposite, nanotubes.

\section{RESUMEN}

En el desarrollo de este trabajo de investigación se han sintetizado y caracterizado nanocompositos en base de halloysita con nanopartículas de sulfuro de bismuto. La halloysita, es un filosilicato que se encuentra en forma de nanotubos de multicapas y constituye una alternativa de morfología similar a los nanotubos de carbono; sin embargo, posee características químicas distintas en la superficie externa e interna. La síntesis se llevó a cabo utilizando un método de impregnación de los precursores en nanotubos de halloysita para el posterior

1 Pontificia Universidad Católica del Ecuador, Facultad de Ciencias Exactas y Naturales, Escuela de Ciencias Químicas, Quito, Ecuador. (*correspondencia: cbvega@puce.edu.ec, carovv25@gmail.com).

2 Universidad Nacional Autónoma de México, Facultad de Química, Departamento de Química Inorgánica y Nuclear, Ciudad de México, México (cgomez.nanoscience@gmail.com) 
crecimiento de las nanopartículas in situ. La caracterización incluyó espectroscopias de absorción electrónica (UV-visible) y difracción de rayos X, en polvos. La morfología de los nanocompositos preparados se evidenció utilizando microscopía de barrido electrónico (SEM) y microscopía de transmisión electrónica (TEM); además se utilizó espectroscopia de energía dispersiva (EDS) para identificar elementos particulares y su distribución en la muestra. Los resultados indican que las nanopartículas de $\mathrm{Bi}_{2} \mathrm{~S}_{3}\left(\mathrm{Bi}_{2} \mathrm{~S}_{3} \mathrm{NPs}\right)$, de morfología esférica, se depositaron de manera uniforme sobre los nanotubos de halloysita (HNTs). Las partículas en el nanocomposito presentaron mayor diámetro que las partículas sintetizadas sin HNTs. Este cambio se evidencia en la reducción del ancho del pico en los patrones de difracción y en la disminución de valor de energía de la brecha energética. La formación del nanocomposito contribuyó a mantener las nanopartículas dispersas de manera homogénea sobre halloysita, evitando su aglomeración. Además, se evidenció el control de tamaño y morfología cuando se utiliza los nanotubos como soporte.

\section{ABSTRACT}

In this work, nanocomposites based on halloysite nanotubes have been synthesized and characterized. Halloysite is a phyllosilicate with multilayer nanotubular structure. Halloysite nanotubes are a low-cost alternative with similar morphology over carbon nanotubes. In addition, the tubular structure is combined with differences in reactivity between the external surface and internal surface. The nanocomposites were synthesized by the impregnation of precursors over halloysite nanotube surface, after that nanoparticle growth was done in situ. The characterization of the nanocomposites included several spectroscopic techniques such as electronic absorption spectroscopy (UV-Vis). Also, $X$-ray diffraction was used for structure characterization. The morphology of the nanocomposites was study with scanning electron microscopy (SEM) and transmission electron microscopy (TEM). In addition, energy dispersive spectroscopy (EDS) was used in order to determine composition and identify distribution of elements over halloysite. The results indicated that $\mathrm{Bi}_{2} \mathrm{~S}_{3}$ nanoparticles $\left(\mathrm{Bi}_{2} \mathrm{~S}_{3}\right.$ NPs) were successfully deposited on the surface of halloysite nanotubes (HNTs). $\mathrm{Bi}_{2} \mathrm{~S}_{3}$ nanoparticles synthesized over halloysite surface have a greater diameter 
than particles synthesized without HNTs as a support; reduction in the peak wide of diffraction patterns and decrement in the bandgap energy value. The development of these nanocomposites account to maintain nanoparticles dispersed all over halloysite and avoiding particle agglomeration. Lastly, halloysite nanotubes contribute to maintain size control and a narrow size distribution of nanoparticles.

\section{INTRODUCCIÓN}

La arcilla halloysita fue detallada por primera vez en 1826 por Berthier, como un mineral 1:1 dioctáedrico. La halloysita es un polimorfo de la caolinita y su fórmula mínima es $\mathrm{Al}_{2} \mathrm{Si}_{2} \mathrm{O}_{5}(\mathrm{OH})_{4}{ }^{*} \mathrm{nH}_{2} \mathrm{O}$ (Hendricks, 1938). Este filosilicato presenta de manera natural varias morfologías, tales como placas o esferas; sin embargo, la morfología predominante es tubular (Singh, 1996). Los nanotubos de halloysita se encuentran dispuestos en multicapas, su preparación no requiere de procesos costosos como la preparación de nanotubos de carbono o de nitruro de boro; esto constituye una alternativa menos costosa que es morfológicamente similar a los nanotubos de carbono (Du, Guo, \& Jia, 2010). Entre las características principales se puede mencionar que la longitud de los nanotubos de haIloysita (HNTs) va de 0,2 a 2,0 $\mu \mathrm{m}$; el diámetro interno se encuentra en el intervalo de $10 \mathrm{~nm}$ a $40 \mathrm{~nm}$ y el diámetro externo de $40 \mathrm{~nm}$ a $70 \mathrm{~nm}$. Además, el tamaño de poro promedio está en el intervalo de 79,7 $\AA$ a $100,2 \AA$ y el tamaño de partícula en solución acuosa al $5 \%$, se encuentra entre los valores de $50 \mathrm{~nm}$ a $400 \mathrm{~nm}$ (Liu, Jia, Jia, \& Zhou, 2014). Materiales nanoestructurados de tipo (1D) como los nanotubos de halloysita son de gran interés debido a su alta resistencia mecánica, estabilidad térmica, biocompatibilidad y abundancia (Liu et al., 2014). Además, son considerados como materiales verdes, ya que su extracción y utilización ocasiona 
un bajo impacto ambiental (Lvov, Wang, Zhang, \& Fakhrullin, 2016).

En la síntesis de nanoestructuras es deseable tener el control de la dispersión, el tamaño y la morfología de partículas, ya que estas variables pueden afectar el desempeño del material en distintas aplicaciones. Se propone el uso de nanotubos de haIloysita (HNTs) como nanoplantillas o nanoreactores para la síntesis de materiales nanoestructurados con distribuciones de tamaño de partícula estrechas (Du et al., 2010). Adicionalmente, la halloysita se propone como componente de materiales avanzados como los nanocompositos, debido a su fácil funcionalización, estructura definida y amplia disponibilidad. Se ha reportado que los nanocompositos $\mathrm{HNTs}_{\mathrm{TiO}}$ presentan mayor actividad fotocatalítica para la degradación de $\mathrm{NO}_{\mathrm{x}}$ bajo luz visible, comparado con $\mathrm{TiO}_{2}$ (P25) comercial. Por lo tanto, los nanotubos de halloysita mejoran la dispersión y actividad fotocatalítica de las nanopartículas de $\mathrm{TiO}_{2}$ (Papoulis et al., 2010).

Al ser un mineral biocompatible, se ha utilizado en la síntesis de nano- compositos HNTs-Ag, tiene buen desempeño en contra de bacterias Gram-negativas como la Escherichia coli y Gram-positiivas como el Staphylococcus aureous (Zhang, Chen, Zhang, Zhang, \& Liu, 2013). Las nanoaleaciones también han sido utilizadas para la formación de compositos con nanotubos de halloysita. Nanoaleaciones de FeNi soportadas en nanotubos de halloysita alcanzan casi $100 \%$ en la descomposición catalítica de $\mathrm{PH}_{3}$ hacia $\mathrm{P}_{4}$ e hidrógeno a baja temperatura (Tang, Li, Shen, \& Wang, 2013).

En el presente trabajo de investigación se describe la síntesis y caracterización de un composito de halloysita con nanopartículas de sulfuro de bismuto, debido a las múltiples aplicaciones de este material. El sulfuro de bismuto $\left(\mathrm{Bi}_{2} \mathrm{~S}_{3}\right)$ es un semiconductor de estructura laminar, la energía de la brecha energética o "bandgap" para la forma microcristalina del material tiene valor de 1,3 eV (Vogel, Hoyer, \& Weller, 1994). Su energía de brecha energética se encuentra en el rango del espectro de luz visible lo que lo hace útil en aparatos de conversión de energía solar (Pejova \& Grozdanov, 2006). Este 
material presenta algunas aplicaciones prácticas tales como dispositivos termoeléctricos (Biswas, Zhao, \& Kanatzidis, 2012), fotodetectores (Konstantatos, Levina, Tang, \& Sargent, 2008), interruptores ópticos (Xi, Hu, Zhang, Zhang, \& Wang, 2009), fotocatálisis para remediación ambiental (Cao, Xu, Lin, Luo, \& Chen, 2012), generación de hidrógeno usando energía solar (Brahimi, Bessekhouad,
Bouguelia, \& Trari, 2007), detector de $\mathrm{H}_{2}$ (Yao et al., 2008), detector de biomoléculas (Cademartiri et al., 2009). Además de sus múltiples aplicaciones es importante señalar que presenta menor impacto ambiental que otros materiales como $\mathrm{PbS}, \mathrm{CdS}$, $\mathrm{HgS}$, que tienen aplicaciones similares (Zumeta, Ortiz, Díaz, Trallero, \& Ruiz, 2014).

\section{MATERIALES Y MÉTODOS}

\section{Materiales y Equipos}

Los reactivos utilizados fueron nitrato de bismuto pentahidratado $\left(\mathrm{Bi}\left(\mathrm{NO}_{3}\right)_{3} \cdot 5 \mathrm{H}_{2} \mathrm{O}\right.$, Sigma, $98 \%$ ), dimetilsulfóxido (DMSO, J.T Baker), azufre elemental (S, Sigma-Aldrich, 99,5 \%), hidróxido de amonio $\left(\mathrm{NH}_{4} \mathrm{OH}\right.$, J.T Baker, 28,3\% de $\left.\mathrm{NH}_{3}\right)$, nanotubos de halloysita $\left(\mathrm{H}_{4} \mathrm{Al}_{2} \mathrm{O}_{9} \mathrm{Si}_{2} \cdot 2 \mathrm{H}_{2} \mathrm{O}\right.$, Sigma), acetona $\left(\mathrm{CH}_{3} \mathrm{COCH}_{3}\right.$, J.T Baker, 99,5 \%) y agua desionizada (resistividad 14 $\mathrm{M} \Omega^{*} \mathrm{~cm}$ ). Todos los reactivos fueron utilizados sin purificación adicional.

Las imágenes TEM y HR-TEM se obtuvieron en un microscopio electró- nico JEOL-2010 con voltaje de 200 $\mathrm{kV}$. El procesamiento de imágenes se realizó utilizando software DigitalMicrograph (GATAN versión 3.7.0). Las imágenes de microscopia electrónica de barrido se obtuvieron en JEOL59 00LV usando voltaje de 20 kV. Los espectros Raman se obtuvieron en EZRaman-N (Enwave Optronics), equipado con un láser de $532 \mathrm{~nm}$. Los patrones de difracción de rayos $X$ (DRX) se midieron con el difractómetro D2Phaser (Bruker) utilizando radiación K $\alpha$ de $\mathrm{Cu}(1,5418 \AA$ ). Los espectros de reflactancia difusa se obtuvieron en Cary-5E Varian; la función de Kubelka-muk se utilizó para estimar el valor de brecha de banda o bandgap. 


\section{Síntesis de NPs de $\mathrm{Bi}_{2} \mathrm{~S}_{3}$}

Se adicionó $1 * 10^{-4} \mathrm{~mol}(49,6 \mathrm{mg})$ de nitrato de bismuto pentahidratado $\mathrm{Bi}\left(\mathrm{NO}_{3}\right)_{3} \cdot 5 \mathrm{H}_{2} \mathrm{O}$ en $40 \mathrm{~mL}$ de DMSO, se calentó la dispersión hasta $120^{\circ} \mathrm{C}$ bajo agitación magnética hasta la completa disolución de la sal precursora de bismuto. Al mismo tiempo se preparó una solución de 4,96 mg de azufre elemental en $10 \mathrm{~mL}$ de DMSO a $120{ }^{\circ} \mathrm{C}$. Se adicionó la solución de azufre a la solución de nitrato de bismuto, finalmente se agregó hidróxido de amonio bajo agitación constante hasta que la solución adquiera una coloración obscura debido al precipitado $\mathrm{Bi}_{2} \mathrm{~S}_{3}$. La suspensión se mantuvo a $120{ }^{\circ} \mathrm{C}$ por 15 minutos. El producto negro se separó mediante centrifugación, se lavó tres veces con agua y tres veces con acetona, se secó a $50{ }^{\circ} \mathrm{C}$ durante 12 horas.

\section{Síntesis del nanocomposito NPs $\mathrm{Bi}_{2} \mathrm{~S}_{3}$-HNTs}

Se adicionó $1 * 10^{-4} \mathrm{~mol}(49,6 \mathrm{mg})$ de $\mathrm{Bi}\left(\mathrm{NO}_{3}\right)_{3} \cdot 5 \mathrm{H}_{2} \mathrm{O}$ y $75 \mathrm{mg}$ de $\mathrm{H}_{4} \mathrm{Al}_{2} \mathrm{O}_{9} \mathrm{Si}_{2} \cdot 2 \mathrm{H}_{2} \mathrm{O}$ (halloysita) en 40 $\mathrm{mL}$ de DMSO. El sistema se mantuvo sellado con parafilm y con agitación magnética, a temperatura ambiente, por 72 horas. Transcurrido el tiempo de impregnación, se calentó la dispersión hasta $120{ }^{\circ} \mathrm{C}$ bajo agitación magnética. En caliente se adicionó una solución de azufre (4,96 mg de azufre elemental en $10 \mathrm{~mL}$ de DM $\mathrm{SO})$, finalmente se agregó $\mathrm{NH}_{4} \mathrm{OH}$ bajo agitación constante hasta que la solución presentó coloración obscura debido al precipitado formado. La suspensión se mantuvo a $120{ }^{\circ} \mathrm{C}$ por 15 minutos. El producto negro se separó mediante centrifugación, se lavó tres veces con agua y tres veces con acetona, se secó a $50{ }^{\circ} \mathrm{C}$ durante 12 horas.

\section{RESULTADOS}

Caracterización de los nanotubos de halloysita

Se llevó a cabo la caracterización de los nanotubos de halloysita utilizando técnicas espectroscópicas y microscopia electrónica. 
La Figura 1 muestra el patrón de difracción (DRX) de halloysita comercial (Sigma Aldrich). Las reflexiones de la estructura cristalina halloysita se caracterizan por líneas relativamente anchas con una pequeña relación de señal a ruido. Además de los picos identificados a partir de halloysita, hay reflejos de las impurezas de feldespato y cuarzo $\left(\mathrm{SiO}_{2}\right)$ a $15,9^{\circ}$ y $26,9^{\circ}$, respectivamente. Las señales de difracción coinciden muy bien con los valores estándar de la Halloy- sita-7 Å (PDF No. 29-1487). Un pico ancho a $12,1^{\circ}$ correspondiente a 7,3 $\AA$ es asignado a las reflexiones basales de primer orden (001). La intercapa de agua en la halloysita (10 ̊) presenta una diferencia de al menos $3 \AA$ en el valor de la señal $d_{001}$ con respecto a halloysita (7 $\AA$ ), esta diferencia representa el grosor de la monocapa de moléculas de agua. El pico de mayor intensidad (100) correspondiente a 4,4 Å y es indicativo de la estructura tubular de la halloysita.

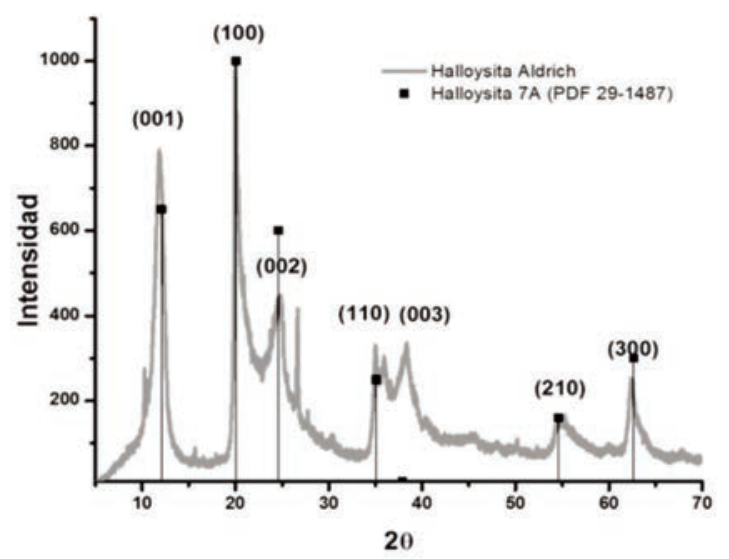

Figura 1. Patrón de difracción de los nanotubos de halloysita

Se realizó un estudio mediante microscopía electrónica de barrido SEM y de transmisión TEM por sus siglas en inglés, para conocer con mayor detalle la morfología de los nanotu- bos. En la Figura 2a se puede observar claramente la morfología tubular de halloysita, la longitud del nanotubo es variable en la muestra. Además, en la Figura $2 b$ es posible 
evidenciar que los nanotubos de halloysita presentan variaciones en su longitud. Adicionalmente, se puede apreciar las secciones internas variables de los nanotubos, las cuáles pue- den ser susceptibles de funciona- lizarse otorgando una matriz para el crecimiento de materiales con morfología tubular.
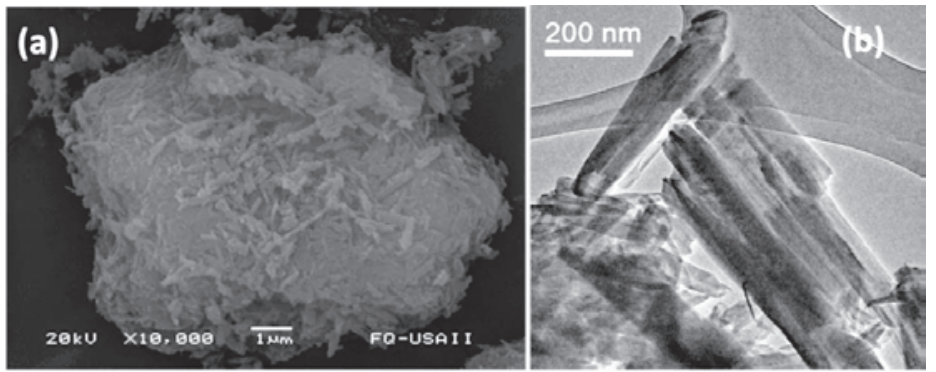

Figura 2. a) Imágenes SEM de nanotubos de halloysita b) Imágenes TEM de nanotubos de halloysita

Caracterización de $\mathrm{NPs} \mathrm{Bi}_{2} \mathrm{~S}_{3}$ y nanocomposito $\mathrm{NPsBi}_{2} \mathrm{~S}_{3}-\mathrm{HNT}$

La estructura de sulfuro de bismuto $\left(\mathrm{Bi}_{2} \mathrm{~S}_{3}\right)$ que se obtiene, posee un sistema cristalino ortorrómbico, con un grupo espacial Pmcn (62). Los parámetros de celda son $\mathrm{a}=3,981(1) \AA$ $\mathrm{b}=11,147(3) \AA \mathrm{c}=11,305(2) \AA$.

En la Figura 3b se muestra el patrón de difracción de las nanopartículas de sulfuro de bismuto (NPs $\mathrm{Bi}_{2} \mathrm{~S}_{3}$ ) sintetizadas, en el cual los picos se encuentran superpuestos formando señales amplias. Este patrón de di- fracción puede justificarse por algunos motivos: a) el orden cristalino es de muy bajo alcance (Zumeta et al., 2014) b) el tamaño muy pequeño de cristalita afecta el ancho de los picos del patrón de difracción. En la figura 3a se observa el patrón de difracción del nanocomposito preparado con sulfuro de bismuto y halloysita. Se puede establecer que las nanopartículas de sulfuro de bismuto presentan un ligero aumento en el diámetro, lo cual resulta en una disminución del ancho de pico de difracción. Adicionalmente, se pueden distinguir los picos con mayor intensidad (001) 
y (100), correspondientes a la fase de halloysita que, a su vez, está aso- ciado a la estructura tubular de la haIloysita.

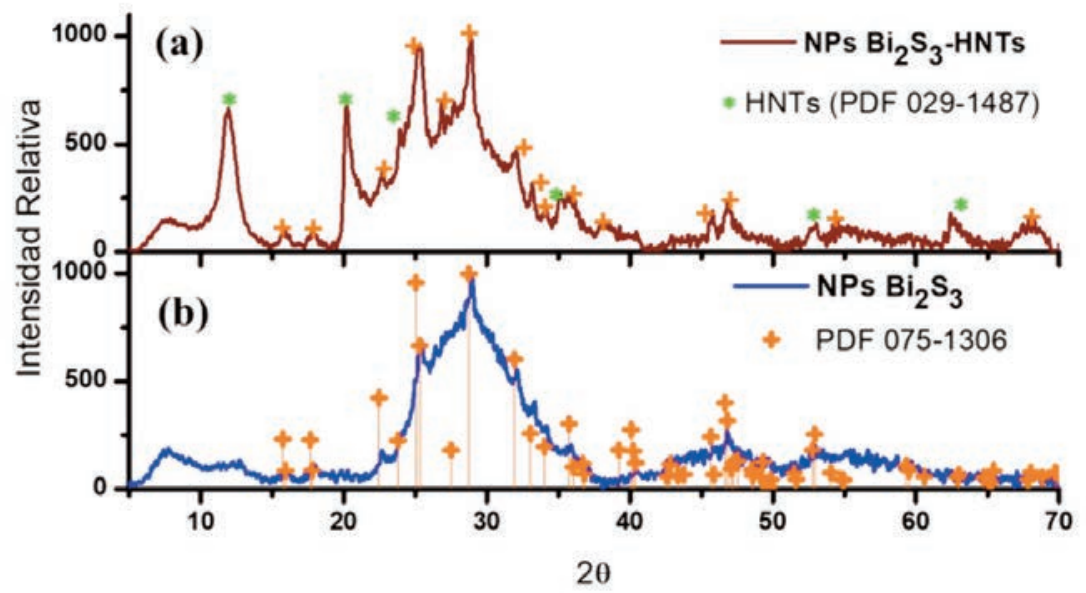

Figura 3. Patrones de difracción de rayos $X$ de a) nanocomposito $\mathrm{NPs} \mathrm{Bi}_{2} \mathrm{~S}_{3}-\mathrm{HNTs}$ b) nanopartículas de $\mathrm{Bi}_{2} \mathrm{~S}_{3}$

En la Figura 4 se observa el espectro de reflactancia difusa de las nanopartículas de sulfuro de bismuto y el nanocomposito con halloysita. Se realizó la estimación de la energía de brecha de banda directa utilizando la gráfica de Tauc. Los valores calculados son distintos entre sí, lo cual se debe al aumento en el tamaño cuando se coloca HNT en la síntesis de NPs $\mathrm{Bi}_{2} \mathrm{~S}_{3}$; adicionalmente la variación en tamaño de partícula está relacionada con el patrón de difracción obtenido para tales sistemas.
En la Figura 5 se muestra los resultados de microscopía SEM-EDS para el nanocomposito $\mathrm{NPS} \mathrm{Bi}_{2} \mathrm{~S}_{3}-\mathrm{HNT}$. El análisis EDS en modo mapeo se utilizó para corroborar que los elementos bismuto y azufre (es decir las NPs $\mathrm{Bi}_{2} \mathrm{~S}_{3}$ ) se encuentran dispersos de manera homogénea en halloysita, la cual está representada por los elementos Al y Si que forman parte de su estructura laminar. 


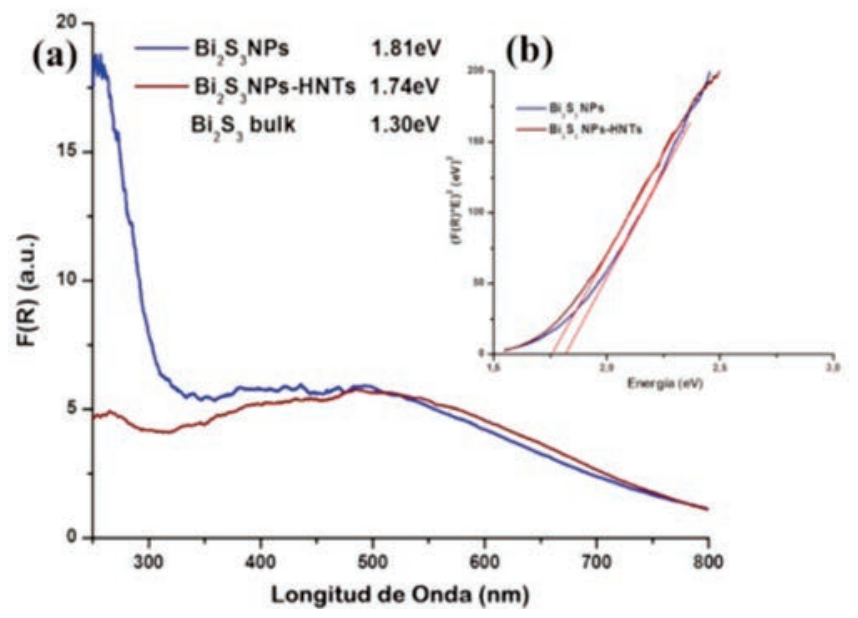

Figura 4. a) Espectro de absorción (modo $F(R)$ ) de nanopartículas $\mathrm{Bi}_{2} \mathrm{~S}_{3}$ y nanocomposito NPs $\mathrm{Bi}_{2} \mathrm{~S}_{3}$-HNT.

b) Estimación de la energía de band-gap directo para ambos materiales
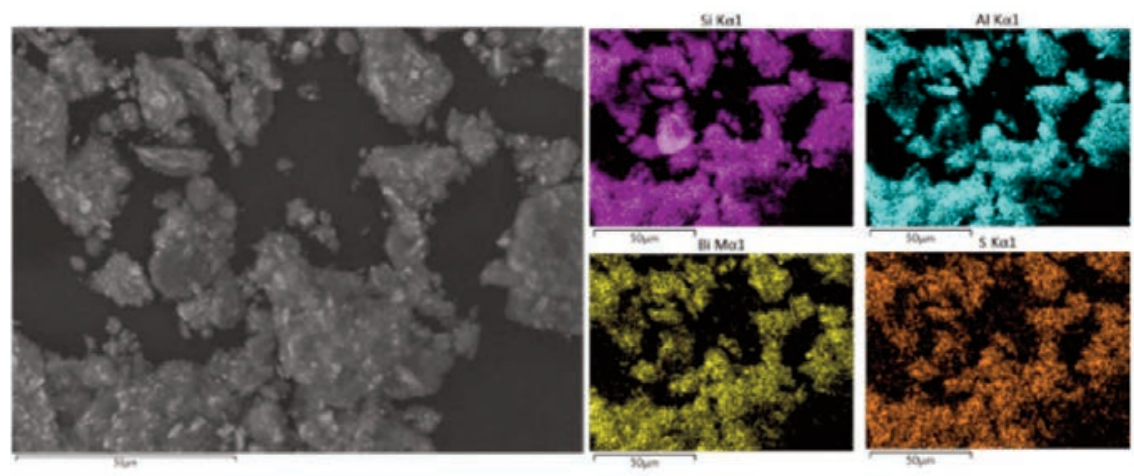

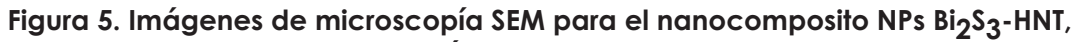
análisis EDS mapeo

En la Figura 6 se observan las imágenes TEM para NPs $\mathrm{Bi}_{2} \mathrm{~S}_{3}$ y el nanocomposito NPs $\mathrm{Bi}_{2} \mathrm{~S}_{3}$-HNT. Es posible diferenciar pequeñas partículas con mayor contraste dispersas sobre los nanotubos de halloysita. 

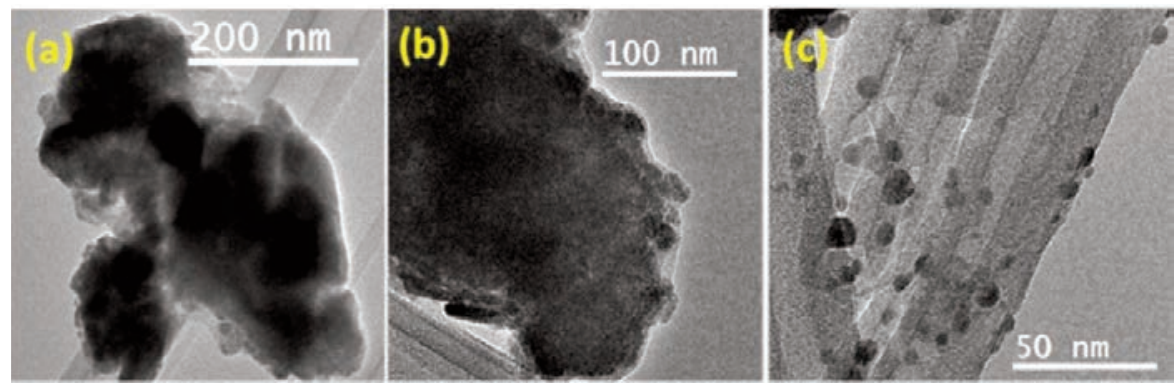

Figura 6. (a) y (b) imágenes TEM de nanopartículas de $\mathrm{Bi}_{2} \mathrm{~S}_{3}$

(c) imágenes TEM de nanocomposito NPs $\mathrm{Bi}_{2} \mathrm{~S}_{3}$-HNTs

\section{DISCUSIÓN}

La síntesis de nanomateriales mediante métodos químicos requiere el control de la concentración de precursores para los procesos de nucleación y crecimiento del material; concentraciones altas de precursores aceleran el crecimiento y pueden modificar la morfología de las nanopartículas (Wu, Zhou, Zhang, \& Zhong, 2010).

La carga negativa en la superficie externa de los nanotubos de halloysita compuesta por grupos siloxano es una propiedad que se aprovechó para la absorción de cationes como $\mathrm{Cu}^{2+}, \mathrm{Bi}^{3+}$ o especies catiónicas polinucleares $\left[\mathrm{Bi}_{6} \mathrm{O}_{4}(\mathrm{OH})_{4}\right]^{6+}$ formadas en agua; de esta manera se logra un crecimiento controlado (morfología y tamaño) de las nanopartículas sintetizadas.

El tamaño de las nanopartículas de sulfuro de bismuto aumenta cuando la síntesis se realiza utilizando nanotubos de halloysita como soporte; este aumento de tamaño se puede relacionar con el tamaño de cristalita, ya que la diminución del ancho de pico en las señales del patrón de difracción refleja un aumento en el tamaño de cristalita (Xing, Feng, Chen, Yao, \& Song, 2003).

El valor de energía de brecha energética para el nanocomposito es 1,74 eV menor al calculado para las nano- 
partículas sin soporte $(1,81 \mathrm{eV})$, esta variación se encuentra relacionada con el cambio en el tamaño de partícula ya que, debido a efectos de confinamiento cuántico, a medida que disminuye el diámetro de partícula se tiene un aumento en la diferencia de energía de la banda de valencia y la banda de conducción del material (Pejova \& Grozdanov, 2006).

Esta variación de tamaño de partícula, se debe a la dispersión adecuada de los precursores sobre los nanotubos favoreciendo la nucleación del material.

Las imágenes de microscopía SEMEDS del nanocomposito permiten establecer que la distribución de $\mathrm{Bi}_{2} \mathrm{~S}_{3}$ es homogénea sobre los nanotubos de halloysita, ya que no se observan diferencias de contraste en la imagen. En el análisis de mapeo EDS se muestra que tanto los elementos bismuto y azufre se encuentran dispersos sobre los nanotubos de halloysita. Con respecto a la morfología, las imágenes HR-TEM permiten distinguir las nanopartículas, así como también evidenciar la función de los nanotubos de halloysita en la notoria disminución de la aglomeración de nanopartículas de sulfuro de bismuto.

Cabe recalcar que el uso de nanotubos de halloysita también puede ocasionar un crecimiento preferencial del material, permitiendo la síntesis de materiales con distintas morfologías. Se ha reportado que el uso de nanotubos de halloysita altera la morfología de las nanopartículas de carbonato de bismutilo de nanoplacas hasta nanoesferas (Ortiz, Vega, Díaz, \& Zumeta, 2018).

\section{CONCLUSIÓN}

Se realizó la caracterización de los nanotubos de halloysita destacando que, debido a su estructura enrollada de multicapas, tienen una cantidad menor de grupos hidroxilo en la superficie externa, esto en comparación con otros filosilicatos y lo que contribuye a la dispersión eficiente de las nanopartículas sintetizadas.

La síntesis del nanocomposito $\mathrm{Bi}_{2} \mathrm{~S}_{3}$ HNT constituye una alternativa para 
la adecuada dispersión de las nanopartículas de sulfuro de bismuto, evitando su aglomeración. La capacidad de modificación del valor de brecha energética en función del tamaño de partícula ofrece mayores ventajas para el uso del material.

\section{LISTA DE REFERENCIAS}

Berthier, P. (1826). Analyse de l'halloysite. Ann.Chim.Phys, 32, 332-335.

Biswas, K., Zhao, L.-D., \& Kanatzidis, M. G. (2012). Tellurium-Free Thermoelectric: The Anisotropic $\mathrm{n}$-Type Semiconductor $\mathrm{Bi}_{2} \mathrm{~S}_{3}$. Advanced Energy Materials, 2(6), 634638. https://doi.org/10.1002/aenm.201100775

Brahimi, R., Bessekhouad, Y., Bouguelia, A., \& Trari, M. (2007). Visible light induced hydrogen evolution over the heterosystem $\mathrm{Bi}_{2} \mathrm{~S}_{3} / \mathrm{TiO}_{2}$. Catalysis Today, 122(1), 6265. https://doi.org/https://doi.org/10.1016/j.cattod.2007.01.030

Cademartiri, L., Scotognella, F., O’Brien, P. G., Lotsch, B. V, Thomson, J., Petrov, S., ... Ozin, G. A. (2009). Cross-Linking $\mathrm{Bi}_{2} \mathrm{~S}_{3}$ Ultrathin Nanowires: A Platform for Nanostructure Formation and Biomolecule Detection. Nano Letters, 9(4), 14821486. https://doi.org/10.1021/nl803417v

Cao, J., Xu, B., Lin, H., Luo, B., \& Chen, S. (2012). Novel $\mathrm{Bi}_{2} \mathrm{~S}_{3}$-sensitized BiOCl with highly visible light photocatalytic activity for the removal of rhodamine B. Catalysis Communications, 26(Supplement C), 204-208. https://doi.org/https://doi.org/10. 1016/j.catcom.2012.05.025

Du, M., Guo, B., \& Jia, D. (2010). Newly emerging applications of halloysite nanotubes: A review. Polymer International. https://doi.org/10.1002/pi.2754

Hendricks, S. (1938). Crystal Structures of the Clay Mineral Hydrates. Nature, 142, 38. doi:10.1038/142038a0 
Konstantatos, G., Levina, L., Tang, J., \& Sargent, E. H. (2008). Sensitive SolutionProcessed $\mathrm{Bi}_{2} \mathrm{~S}_{3}$ Nanocrystalline Photodetectors. Nano Letters, 8(11), 4002-4006. https://doi.org/10.1021/nl802600z

Liu, M., Jia, Z., Jia, D., \& Zhou, C. (2014). Recent advance in research on halloysite nanotubes-polymer nanocomposite. Progress in Polymer Science, 39(8), 1498-1525. https://doi.org/https://doi.org/10.1016/j.progpolymsci.2014.04.004

Lvov, Y., Wang, W., Zhang, L., \& Fakhrullin, R. (2016). Halloysite Clay Nanotubes for Loading and Sustained Release of Functional Compounds. Advanced Materials, 28(6), 1227-1250. https://doi.org/10.1002/adma.201502341

Ortiz, J. L., Vega, C., Díaz, D., \& Zumeta, I. (2018). Transformation of Bismuth and $\beta$ Bi2O3 Nanoparticles into $(\mathrm{BiO})_{2} \mathrm{CO}_{3}$ and $(\mathrm{BiO})_{4}(\mathrm{OH})_{2} \mathrm{CO}_{3}$ by Capturing $\mathrm{CO}_{2}$ : The Role of Halloysite Nanotubes and "Sunlight" on the Crystal Shape and Size. Crystal Growth \& Design, 18(8), 4334-4346. https://doi.org/10.1021/acs. cgd.8b00177

Papoulis, D., Komarneni, S., Nikolopoulou, A., Tsolis-Katagas, P., Panagiotaras, D., Kacandes, H. G.,Katsuki, H. (2010). Palygorskite- and Halloysite- $\mathrm{TiO}_{2}$ nanocomposites: Synthesis and photocatalytic activity. Applied Clay Science, 50(1), 118-124. https://doi.org/https://doi.org/10.1016/j.clay.2010.07.013

Pejova, B., \& Grozdanov, I. (2006). Structural and optical properties of chemically deposited thin films of quantum-sized bismuth(III) sulfide. Materials Chemistry and Physics, 99(1), 39-49. https://doi.org/10.1016/j.matchemphys.2005.10.010

Singh, B. (1996). Why Does Halloysite Roll?_A New Model. Clays and Clay Minerals, 44(2), 191-196. https://doi.org/10.1346/CCMN.1996.0440204

Tang, X., Li, L., Shen, B., \& Wang, C. (2013). Halloysite-nanotubes supported FeNi alloy nanoparticles for catalytic decomposition of toxic phosphine gas into yellow phosphorus and hydrogen. Chemosphere, 91(9), 1368-1373. https://doi.org/https://doi. org/10.1016/j.chemosphere.2013.02.010 
Vogel, R., Hoyer, P., \& Weller, H. (1994). Quantum-Sized PbS, CdS, Ag $\mathrm{S}_{2} \mathrm{Sb}_{2} \mathrm{~S}_{3}$, and $\mathrm{Bi}_{2} \mathrm{~S}_{3}$ Particles as Sensitizers for Various Nanoporous Wide-Bandgap Semiconductors. The Journal of Physical Chemistry, 98(12), 3183-3188. https://doi.org/10. 1021/j100063a022

Wu, T., Zhou, X., Zhang, H., \& Zhong, X. (2010). Bi ${ }_{2} \mathrm{~S}_{3}$ nanostructures: A new photocatalyst. Nano Research, 3(5), 379-386. https://doi.org/10.1007/s12274-0101042-0

Xi, Y., Hu, C., Zhang, X., Zhang, Y., \& Wang, Z. L. (2009). Optical switches based on $\mathrm{Bi}_{2} \mathrm{~S}_{3}$ nanowires synthesized by molten salt solvent method. Solid State Communications, 149(43), 1894-1896. https://doi.org/https://doi.org/10.1016/j.ssc.2009. 08.003

Xing, G., Feng, Z., Chen, G., Yao, W., \& Song, X. (2003). Preparation of different morphologies of nanostructured bismuth sulfide with different methods. Materials Letters, 57(29), 4555-4559. https://doi.org/https://doi.org/10.1016/S0167-577X (03)00361-6

Yao, K., Gong, W. W., Hu, Y. F., Liang, X. L., Chen, Q., \& Peng, L.-M. (2008). Individual $\mathrm{Bi}_{2} \mathrm{~S}_{3}$ Nanowire-Based Room-Temperature $\mathrm{H}_{2}$ Sensor. The Journal of Physical Chemistry C, 112(23), 8721-8724. https://doi.org/10.1021/jp8022293

Zhang, Y., Chen, Y., Zhang, H., Zhang, B., \& Liu, J. (2013). Potent antibacterial activity of a novel silver nanoparticle-halloysite nanotube nanocomposite powder. Journal of Inorganic Biochemistry, 118(Supplement C), 59-64. https://doi.org/https://doi. org/10.1016/j.jinorgbio.2012.07.025

Zumeta, I., Ortiz, J. L., Díaz, D., Trallero, C., \& Ruiz, V. F. (2014). First order raman scattering in bulk $\mathrm{Bi}_{2} \mathrm{~S}_{3}$ and quantum dots: Reconsidering controversial interpretations. Journal of Physical Chemistry C, 118(51), 30244-30252. https://doi.org/10.1021/jp509636n 